\title{
Lipid and antioxidant changes in semen of broiler fowl from 25 to 60 weeks of age
}

\author{
K. A. Kelso ${ }^{1}$, S. Cerolini' ${ }^{2}$, R. C. Noble ${ }^{1}$, N. H. C. Sparks ${ }^{1}$ and \\ B. K. Speake ${ }^{1}$ \\ ${ }^{1}$ Department of Biochemical Sciences, Scottish Agricultural College, Auchincruive, Ayr, KA6 5HW, UK, \\ and ${ }^{2}$ Istituto per la Difesa e la Valorizzazione del Germoplasma Animale, Consiglio Nazionale delle \\ Ricerche, via Celoria 10, 20133 Milano, Italy
}

\begin{abstract}
Spermatozoa and seminal plasma from cockerels at the beginning and end of their reproductive periods were examined for their lipid composition and associated antioxidant capacities. The significant reduction in concentration of spermatozoa with age was associated with a large increase in lipid concentrations in spermatozoa and in seminal plasma. This change in lipid concentration was accompanied by increases in the proportions of phospholipid and free cholesterol; in constrast, the proportions of these lipid moieties in seminal plasma were reduced. The major phospholipid fractions in the spermatozoa and seminal plasma were phosphatidyl choline and phosphatidyl ethanolamine. There was a large decrease with age in the proportion of phosphatidyl ethanolamine and a commensurate increase in that of phosphatidyl choline in the spermatozoa and seminal plasma. These major changes in phospholipid content were accompanied by a decrease in the amount of phosphatidyl serine in the spermatozoa and increases in phosphatidyl inositol and cardiolipin in both spermatozoa and seminal plasma. The reductions in the proportions of phosphatidyl ethanolamine were accompanied by considerable reductions in the content of the major polyunsaturated fatty acids 20:4 (n-6) and 22:4 (n-6). The changes in lipid composition owing to ageing were associated with a marked reduction within the spermatozoa of the major antioxidant enzyme glutathione peroxidase. The role of these changes in the specific combinations of polyunsaturated lipids and in antioxidant capacity in the reduction in fertility with age are discussed.
\end{abstract}

\section{Introduction}

A striking feature of spermatozoa from all animal species is the presence of extremely high concentrations of polyunsaturated phospholipids (Neill and Masters, 1972; Poulos et al., 1973; Poulos and White, 1973; Scott, 1973; Lin et al., 1993). In mammalian species, the principal polyunsaturate present is docosahexaenoic acid (22:6 n-3) (Neill and Masters, 1972; Poulos et al., 1973; Evans and Setchell, 1978; Nissen and Kreysel, 1983; Lin et al., 1993). For example, in bulls, docosahexaenoic acid accounts for about $55 \%$ of the total phospholipid fatty acids, with particularly high concentrations occurring within the phosphatidyl ethanolamine and phosphatidyl choline fractions (Jain and Anand, 1976). In contrast, avian spermatozoa exhibit very low concentrations of docosahexaenoic acid and n-3 fatty acids. However, this is compensated for by the presence of substantial concentrations within the phospholipids of $\mathrm{C} 20$ and $\mathrm{C} 22$ polyunsaturates of the $n-6$ series, in particular, arachidonic (20:4) and docosatetra-

Revised manuscript received 17 August 1995

enoic (22:4) acids (Darin-Bennett et al., 1974; Howarth et al., 1977; Ravie and Lake, 1985). There is considerable evidence that the lipid composition of the sperm membrane is a major determinant of motility, cold sensitivity and overall viability (Bakst and Sexton, 1979; Elias et al., 1979; Bearer and Friend, 1982; Hammerstedt, 1993; Roldan and Harrison, 1993). The presence of such high concentrations of polyunsaturated fatty acids within the lipid fractions necessitates the presence of an efficient antioxidant system to protect against peroxidative damage and possible associated sperm dysfunction (Jones $e t$ al., 1979; Wishart, 1984; Alvarez and Storey, 1989; Selley et al, 1991; Cecil and Bakst, 1993; Aitken, 1994). A combination of optimal phospholipid fatty acid composition and antioxidative protection may therefore be envisaged as a major determinant of male fertility.

The present study was undertaken to investigate the relationship between changes in some indicators of semen quality and the nature of the lipids, their fatty acid composition and the activities of the antioxidant enzymes glutathione peroxidase and superoxide dismutase in semen samples from cockerels during ageing. 


\section{Materials and Methods}

\section{Birds and semen collection}

Two groups each of 12 male, naked neck, broiler breeders selected for meat production from the Poultry Science Department (SAC) were used. All males were from the same genetic stock and had hatched at the Poultry Science Department. The males in group 1 were 25 weeks old and in group 2 were 60 weeks old at the beginning of the experiment. Males were housed in single cages in a controlled environment under a photoperiod of $14 \mathrm{~h}$ light: $10 \mathrm{~h}$ dark. All birds were fed $120 \mathrm{~g}$ day $^{-1}$ of a standard commercial diet with $17 \%$ crude protein and $11.5 \mathrm{MJ} \mathrm{kg}^{-1}$ of metabolizable energy. Regular lipid analysis of the feed was undertaken to establish the lipid and fatty acid composition. Semen samples were collected from all birds twice a week throughout the experimental period and three times a week for 3 weeks for laboratory analysis by the method of Lake and Stewart (1978).

\section{Evaluation of spermatozoa}

Within 20 min of collection, the following semen parameters were measured for each male: ejaculate volume (using a graduated tube); sperm concentration (spectrophotometrically) (Brillard and McDaniel, 1985), live spermatozoa (eosin/nigrosin staining) (El Jack and Lake, 1966) and metabolic activity of spermatozoa (Chaudhuri and Wishart, 1988). A portion of fresh semen was used to assay the antioxidant enzymes, superoxide dismutase and glutathione peroxidase.

\section{Preparation of semen for lipid extraction}

After the in vitro tests, the remaining semen was measured and diluted with an equal volume of $0.85 \%(\mathrm{w} / \mathrm{v}) \mathrm{NaCl}$ solution and centrifuged at $700 \mathrm{~g}$ for $20 \mathrm{~min}$ at $4{ }^{\circ} \mathrm{C}$. The upper diluted plasma layer was tranferred to a fresh test tube, the wash procedure was repeated with $1 \mathrm{ml}$ of $0.85 \%(\mathrm{w} / \mathrm{v}) \mathrm{NaCl}$ and the final cell pellet was resuspended in $2 \mathrm{ml} 0.85 \%(\mathrm{w} / \mathrm{v}) \mathrm{NaCl}$. The successive samples obtained during the 3 week collection period were combined for each male to obtain sufficient material for analysis from each bird.

\section{Lipid analysis}

Total lipid was extracted from the spermatozoa and seminal plasma preparations after homogenization in a suitable excess of chloroform:methanol (2:1 v:v) (Christie, 1982). The lipids were fractionated into their major classes (phospholipid, free cholesterol, triacylglycerol, free fatty acids and cholesterol ester) by thin layer chromatography on silica gel G using a solvent system of hexane:diethyl ether:formic acid (80:20:1 $\mathrm{v}: \mathrm{v}: \mathrm{v})$. After visualization under UV light by spraying with $0.1 \%$ (w:v) 2,7-dichlorofluorescein in methanol, the separated bands were scraped from the plates. Phospholipid was eluted from the silica by washing three times with $2 \mathrm{ml}$ methanol and the other lipid classes were similarly eluted with diethyl ether. The esterified lipid fractions were subjected to transmethylation by refluxing with methanol:toluene:sulfuric acid $(20: 10: 1 \quad \mathrm{~V}: \mathrm{v}: \mathrm{v})$ in the presence of a pentadecaenoic acid standard (Christie et al., 1970). The resultant fatty acid methyl esters were analysed by a $1 \mu \mathrm{l}$ injection, via a CP9010 autosampler (Chrompack, London), onto a $30 \mathrm{~m} \times 0.25 \mathrm{~mm}$ diameter, $0.25 \mu \mathrm{m}$ film thickness, Carbowax capillary column (Econo-Cap: Alltech UK Ltd, Carnforth) fitted to a Chrompack CP9001 instrument (Chrompack). Integration of the peaks using an 'EZ-Chrom' Data Handling System (Speck Analytical, Alloa) enabled the determination of the fatty acid composition (\% $w / w$ of total fatty acids). The amount of each lipid class was calculated by comparing the total fatty acid peak areas with that of the pentadecaenoic fatty acid standard (Christie et al., 1970). Free cholesterol was determined by standard colorimetric assay (Boehringer, Lewes).

Individual phospholipid classes were separated by high performance thin layer chromatography (HPTLC) using a solvent system of methyl acetate:isopropanol:chloroform: methanol:0.25\% ( $w / v$ in $\left.\mathrm{H}_{2} \mathrm{O}\right) \mathrm{KCl}(25: 25: 25: 10: 9$, v:v:v:v:v), as described by Olsen and Henderson (1989). After charring (bands were darkened by putting plates in an oven at $160^{\circ} \mathrm{C}$ for 20 min after spraying with $3 \%(\mathrm{w}: \mathrm{v})$ cupric acetate: $8 \%(\mathrm{v}: \mathrm{v})$ orthophosphoric acid), quantification was performed by densitometry using a Shimadzu CS-900I PC dual wavelength flying spot thin layer scanner (Dyson Instruments, Houghton Le Spring, UK). Purification of phosphatidyl choline and phosphatidyl ethanolamine from sperm cells before fatty acid analysis was performed by HPLC according to the method described by Christie (1988).

\section{Enzyme assays}

The assays were performed on a portion of the fresh semen samples within $20 \mathrm{~min}$ of collection, using kits (Randox Limited, Crumlin). The glutathione peroxidase assay is based on the enzyme-catalysed oxidation of reduced glutathione by cumene hydroperoxide coupled to the reduction of the oxidized glutathione by NADPH. Units of glutathione peroxidase activity are expressed as $\mu \mathrm{mol}$ NADPH oxidized $\min ^{-1}$. The superoxide dismutase assay uses xanthine and xanthine oxidase to generate superoxide radicals, which are detected via formazan dye formation. Units of superoxide dismutase activity are defined as the amount of superoxide dismutase required to inhibit the rate of formation of formazan dye by $50 \%$, under the conditions specified by the manufacturers of the kit.

\section{Statistical analyses}

Student's t-test was used for statistical comparison of the data from the two experimental groups. These included 12 samples per group for lipid analysis from ejaculates pooled from three collections, 12 from each group for superoxide dismutase, glutathione peroxidase and metabolic activity, with 36 samples per group for all other analyses including sperm concentration and percentage of live spermatozoa.

\section{Results}

The characteristics of the semen samples from the young and old donors, in terms of number of spermatozoa, proportion of live cells, metabolic activity and antioxidant enzyme activity Downloaded from Bioscientifica.com at 04/26/2023 07:40:58AM 
Table 1. Semen characteristics of young and old cockerels

\begin{tabular}{lrr}
\hline & \multicolumn{2}{c}{ Age of birds (weeks) } \\
\cline { 2 - 3 } Parameter & \multicolumn{1}{c}{25} & 60 \\
\hline Sperm concentration $\left(10^{9}\right.$ cells ml $^{-1}$ ) & & $1.08 \pm 0.41$ \\
Live spermatozoa (\%) & $91.20 \pm 3.60$ & $86.09 \pm 7.82^{\mathrm{a}}$ \\
Metabolic activity (nmol formazan dye reduced per $10^{9}$ cells per min) & $0.24 \pm 0.08$ & $0.06 \pm 0.02^{\mathrm{a}}$ \\
Glutathione peroxidase (units per $10^{9}$ cells) & $2.53 \pm 0.31$ & $0.23 \pm 0.09^{\mathrm{a}}$ \\
Superoxide dismutase (units per $10^{9}$ cells) & $0.24 \pm 0.12$ & $0.17 \pm 0.06$ \\
\hline
\end{tabular}

Values are means \pm SD of individual observations from 12 birds in each age group.

Significance of difference between age groups: ${ }^{\circ} P<0.001$.

Table 2. Lipid composition of spermatozoa and seminal plasma from young and old cockerels

\begin{tabular}{|c|c|c|c|c|c|c|c|c|}
\hline \multirow{2}{*}{$\begin{array}{l}\text { Age of donors } \\
\text { (weeks) }\end{array}$} & \multicolumn{4}{|c|}{ Spermatozoa } & \multicolumn{4}{|c|}{ Seminal plasma } \\
\hline & \multicolumn{2}{|l|}{25} & \multicolumn{2}{|l|}{60} & \multicolumn{2}{|l|}{25} & \multicolumn{2}{|l|}{60} \\
\hline $\begin{array}{l}\text { Total lipid ( } \mu \text { g per } 10^{\circ} \text { spermatozoa } \\
\quad \text { or } \mu \mathrm{g} \mathrm{ml}^{-1} \text { seminal plasma) }\end{array}$ & \multicolumn{2}{|c|}{$574.55 \pm 111.45$} & \multicolumn{2}{|c|}{$1122.44 \pm 307.07^{\mathrm{a}}$} & \multicolumn{2}{|c|}{$142.76 \pm 29.95$} & \multicolumn{2}{|c|}{$1104.03 \pm 775.30^{\mathrm{b}}$} \\
\hline \multicolumn{9}{|l|}{ Lipid class (\% w/w of total lipid) } \\
\hline Phospholipid & $66.20 \pm$ & 4.11 & $72.09 \pm$ & $5.40^{c}$ & $34.17 \pm$ & 4.79 & $28.39 \pm$ & 4.98 \\
\hline Free cholesterol & $18.52 \pm$ & 2.61 & $20.16 \pm$ & 5.05 & $19.89 \pm$ & 7.68 & $16.02 \pm$ & 4.28 \\
\hline Triacylglycerol & $9.73 \pm$ & 3.15 & $2.20 \pm$ & $1.86^{\mathrm{a}}$ & $22.02 \pm$ & 7.61 & $20.08 \pm$ & 8.40 \\
\hline Free fatty acid & nd & & $3.96 \pm$ & $1.89^{a}$ & $12.97 \pm$ & 5.27 & $14.33 \pm$ & 4.20 \\
\hline Cholesterol ester & $5.62 \pm$ & 1.96 & $2.42 \pm$ & $1.44^{c}$ & $10.97 \pm$ & 4.26 & $20.76 \pm$ & $9.83^{\mathrm{c}}$ \\
\hline
\end{tabular}

Values are means \pm SD of individual observations from 12 birds in each age group.

Significance of difference between age groups: ${ }^{a} P<0.001$; ${ }^{b} P<0.01 ;{ }^{c} P<0.05$.

nd: not detectable.

are given (Table 1). The concentration of spermatozoa and the proportion of live cells in the semen samples from the older birds was significantly lower than that observed for the younger age group. The metabolic activity of spermatozoa from the oider donors was significantly reduced, being only $25 \%$ of that for the young birds. There was a marked decrease with age (approximately ten-fold) in the activity of glutathione peroxidase of spermatozoa but no change in the activity of superoxide dismutase.

Total lipid concentrations and proportions (expressed as a percentage of total lipids) of the major lipid classes present in the spermatozoa and seminal plasma of the young and old donors are given (Table 2). There was a wide variation in the total lipid concentrations in the spermatozoa and in seminal plasma; however, in both fractions, lipid concentrations for the older donors were significantly greater than for the younger donors. Phospholipid was the predominant lipid fraction within the spermatozoa and was accompanied by substantial amounts of free cholesterol. The concentration of phospholipid in the seminal plasma was considerably lower than in the spermatozoa but concentrations of cholesterol ester, triacylglycerol and free fatty acid were much higher. Although, there was an overall similarity in lipid composition between the two groups, spermatozoa from the older donors showed significant differences in all the lipid classes other than free cholesterol and, in contrast to the young donors, free fatty acids were detectable.
The difference in the lipid composition of the seminal plasma between the young and old donors was minimal.

The proportions of the major phospholipid fractions (as a percentage of total phospholipid) in the spermatozoa and seminal plasma of the young and old donors are given (Table 3). The major phospholipid fractions in the spermatozoa of the young donors were phosphatidyl ethanolamine and phosphatidyl choline accompanied by substantial amounts of phosphatidyl serine. In the spermatozoa of the older donors, there was a considerable reduction in the amounts of the phosphatidyl ethanolamine and phosphatidyl serine and a concomitant increase in the amounts of phosphatidyl choline, in particular, and phosphatidyl inositol. Phosphatidyl ethanolamine was the major lipid fraction in the seminal plasma of the young donors. The pattern of change with age was similar to that displayed by the spermatozoa, namely a large reduction in the proportion of phosphatidyl ethanolamine and increases in phosphatidyl choline and phosphatidyl inositol. Cardiolipin was not detectable in either the spermatozoa or the seminal plasma of young donors; however, it contributed a substantial proportion of the total phospholipid in older donors.

The major carriers of the polyunsaturated fatty acids in the spermatozoa of the young and the older donors were the phosphatidyl ethanolamine and phosphatidyl choline fractions. The major polyunsaturates in both the phosphatidyl ethanolamine and phosphatidyl choline were arachidonic acid (20:4) 
Table 3. Phospholipid classes of spermatozoa and seminal plasma from young and old cockerels

\begin{tabular}{|c|c|c|c|c|}
\hline \multirow{2}{*}{$\begin{array}{l}\text { Age of donors } \\
\text { (weeks) }\end{array}$} & \multicolumn{2}{|c|}{ Spermatozoa } & \multicolumn{2}{|c|}{ Seminal plasma } \\
\hline & 25 & 60 & 25 & 60 \\
\hline \multicolumn{5}{|c|}{ Phospholipid fraction, (\% w/w of total phospholipid) } \\
\hline Phosphatidyl choline & $31.74 \pm 3.25$ & $46.53 \pm 6.03^{\mathrm{a}}$ & $9.84 \pm 1.89$ & $21.31 \pm 4.35^{\mathrm{b}}$ \\
\hline Phosphatidyl ethanolamine & $30.33 \pm 1.91$ & $17.20 \pm 7.18^{\mathrm{a}}$ & $52.60 \pm 3.94$ & $34.58 \pm 1.92^{b}$ \\
\hline Phosphatidyl serine & $24.04 \pm 3.62$ & $5.91 \pm 4.99^{\mathrm{a}}$ & $18.16 \pm 3.52$ & $14.67 \pm 3.99$ \\
\hline Phosphatidyl inositol & $3.56 \pm 0.89$ & $10.01 \pm 5.47^{\mathrm{b}}$ & nd & $10.59 \pm 3.26^{\mathrm{a}}$ \\
\hline Sphingomyelin & $10.32 \pm 2.63$ & $9.60 \pm 4.53$ & $19.40 \pm 3.49$ & $7.54 \pm 5.20^{c}$ \\
\hline Cardiolipin & nd & $10.74 \pm 5.63^{\mathrm{a}}$ & nd & $11.31 \pm 1.82^{\mathrm{a}}$ \\
\hline
\end{tabular}

Values are means \pm SD of individual observations from 12 birds in each age group.

Significance of difference between age groups: ${ }^{a} P<0.001 ;{ }^{b} P<0.01 ;{ }^{c} P<0.05$.

nd: not detectable.

Table 4. Fatty acid composition of the phosphatidyl ethanolamine and phosphatidyl choline fractions of the spermatozoa phospholipids from young and older cockerels

\begin{tabular}{|c|c|c|c|c|}
\hline \multirow{2}{*}{$\begin{array}{l}\text { Age of donors } \\
\text { (weeks) }\end{array}$} & \multicolumn{2}{|c|}{ Phosphatidyl ethanolamine } & \multicolumn{2}{|c|}{ Phosphatidyl choline } \\
\hline & 25 & 60 & 25 & 60 \\
\hline \multicolumn{5}{|c|}{ Fatty acid $(\% \mathrm{w} / \mathrm{w})$} \\
\hline $16: 0$ & $8.10 \pm 1.71$ & $9.71 \pm 1.77$ & $23.08 \pm 1.57$ & $21.42 \pm 4.24$ \\
\hline $18: 0$ & $14.68 \pm 0.57$ & $10.51 \pm 2.28$ & $31.97 \pm 2.17$ & $32.30 \pm 3.47$ \\
\hline $18: 1(n-9)$ & $7.98 \pm 0.34$ & $10.54 \pm 2.83$ & $16.76 \pm 1.59$ & $13.93 \pm 2.34^{c}$ \\
\hline $20: 1$ & $6.83 \pm 0.29$ & $4.23 \pm 2.07$ & $4.56 \pm 0.38$ & $4.72 \pm 0.67$ \\
\hline $20: 4(n-6)$ & $28.91 \pm 4.14$ & $13.99 \pm 1.24^{b}$ & $9.74 \pm 0.88$ & $9.57 \pm 1.64$ \\
\hline $20: 5(n-3)$ & nd & $6.19 \pm 1.22^{b}$ & nd & nd \\
\hline $22: 4(n-6)$ & $33.49 \pm 5.26$ & $23.09 \pm 0.25^{\mathrm{c}}$ & $12.35 \pm 0.49$ & $15.20 \pm 2.84^{\circ}$ \\
\hline $22: 6(n-3)$ & nd & $5.79 \pm 2.02^{c}$ & nd & nd \\
\hline
\end{tabular}

Values are means \pm SD of individual observations from 12 birds in each age group.

Significance of difference between age groups: ${ }^{a} P<0.001 ;{ }^{b} P<0.01 ;{ }^{c} P<0.05$.

nd: not detectable.

and docosatetraenoic acid (22:4) (Table 4). Together these two acids accounted for some $62 \%$ of the total fatty acid present within the phosphatidyl ethanolamine fraction of young donors compared with a total value of $23 \%$ in the phosphatidyl choline fraction. The phosphatidyl choline fraction was characterized by a very much higher proportion of palmitic (16:0) and stearic (18:0) acids. The major difference between the two groups of birds was the very large reduction that occurred in the amounts of arachidonic and docosatetraenoic acids within the phosphatidyl ethanolamine fraction of the spermatozoa with age. The amounts of arachidonic and docosatetraenoic acid in the phosphatidyl ethanolamine fraction of spermatozoa from older birds were reduced by approximately $50 \%$ and $35 \%$, respectively, in comparison with young donors. The fatty acid composition of the phosphatidyl choline fraction of the spermatozoa did not exhibit similar major, age-related changes.

\section{Discussion}

In general, the composition and specific lipid and fatty acid features of the spermatozoa and seminal plasma displayed in the young donors in the present study were similar to those reported previously for sexually active domestic birds (Darin-Bennett et al., 1974; Howarth et al., 1977; Howarth, 1980; Ravie and Lake, 1985). As in other animal species, the lipids of the spermatozoa and also to a large extent those of the seminal plasma, were dominated by polyunsaturated phospholipids, comprising mainly phosphatidyl ethanolamine and phosphatidyl choline. In common with mammals, the spermatozoa and the seminal plasma in the birds used in the present study were characterized by the presence of very high proportions of $\mathrm{C} 20$ and $\mathrm{C} 22$ polyunsaturates. However, the major polyunsaturate present was docosatetraenoic acid $(22: 4 \mathrm{n}-6)$, in contrast to mammals in which docosahexaenoic acid (22:6 n-3) predominates (Neill and Masters, 1972; Poulos et al., 1973; Darin-Bennett et al., 1974; Howarth et al., 1977; Evans and Setchell, 1978; Nissen and Kreysel, 1983; Ravie and Lake, 1985; Lin et al., 1993). The only other tissues that express such high proportions of 22:6n-3 are the brain and retina in which this fatty acid has been shown to play an essential role in development and function (Neuringer et al., 1988). 
A feature of the spermatozoa and seminal plasma of the older birds was the considerable enhancement in gross lipid content accompanied by significant changes in neutral lipid composition. Both endogenous and exogenous lipids play a substantial role in the supply of energy for the motility and viability of spermatozoa (Scott, 1973). The presence of high proportions of neutral lipids within the seminal plasma of a range of animal species may indicate their potential as an energy source. In common with the present findings, a characteristic of decreased fertility in men is a higher proportion of lipid within the cellular and fluid components of semen (Sebastian et al., 1987). In the study reported here, adverse changes in the quality of spermatozoa with age were associated with a similar increase in the lipid content of the semen. It appears, therefore, that an effect of ageing is a change in the use of endogenous and exogenous lipid sources, by spermatozoa.

The major finding of the present investigations associated with the different ages of the donors was the highly significant reduction with age in the phosphatidyl ethanolamine fraction within spermatozoa and their specific content of arachidonic and docosatetraenoic acids. In addition, there was an associated alteration with age in the proportion of other phospholipid classes in both the cellular and plasma components. In particular, the highly unsaturated phosphatidyl ethanolamine was replaced by the more saturated phosphatidyl choline.

There is considerable evidence from other animal species that the lipid composition of sperm membranes may play a key role in determining a range of features associated with overall fertility, both in fresh ejaculates (through an effect on motility) and in stored ejaculates (through an effect on cold sensitivity) (Darin-Bennett et al., 1974; Nissen et al., 1981; Nissen and Kreysel, 1983; Wishart, 1994; Ravie and Lake, 1985). These functional roles of lipids, particularly in association with their highly polyunsaturated fatty acid content, may be related to the biophysical properties of the sperm membrane such as fluidity and permeability (Hammerstedt, 1993). More specific roles such as in membrane fusion (Bearer and Friend, 1982), leukotriene production (Oliw and Sprecher, 1989) and signal transduction (Roldan and Harrison, 1993) have been implicated in the acrosome reaction and fertilization process.

The causes of the changes in lipid composition observed between the two groups of birds in the present study can only be speculated upon. The birds were maintained on identical diets throughout the investigation; therefore, differences due to diet are unlikely. One possible explanation is a significant reduction in the capacity of testicular enzymes to synthesize $\mathrm{C} 20$ and $\mathrm{C} 22$ polyunsaturates or a failure to incorporate the polyunsaturates into the phospholipid fractions during differentiation of the spermatozoa. However, a requirement of cells that display high amounts of polyunsaturated fatty acids is an efficient antioxidant system for protection against peroxidative damage (Froman and Thurston, 1981; Hammerstedt, 1993). Lipid peroxidation has been implicated in spermatozoa dysfunction resulting from structural damage of the plasma membrane in both fresh ejaculates and following storage Jones et al., 1979; Wishart, 1984; Alvarez and Storey, 1989; Selley et al., 1991; Cecil and Bakst, 1993). It is possible that attainment of maximum fertility potential requires a combination of optimal phospholipid fatty acid composition together with appropriate antioxidant protection. It is, therefore, of significance that in the present study the major changes in the phospholipid composition and associated reduction in amounts of polyunsaturates with age of donor were accompanied by a marked reduction in the activities of the antioxidant enzyme glutathione peroxidase.

The authors are grateful to the Scottish Office Agriculture and Fisheries Department for financial support and to the University of Glasgow for the William Stewart Scholarship to K. A. Kelso.

\section{References}

Aitken RJ (1994) A free radical theory of male infertility Reproduction Fertility and Development 6 19-24

Alvarez JG and Storey BT (1989) Role of glutathione peroxidase in protecting mammalian spermatozoa from loss of motility caused by spontaneous lipid peroxidation Gamete Research 23 77-90

Bakst MR and Sexton TJ (1979) Fertilising capacity and ultrastructure of fowl and turkey spermatozoa before and after freezing Journal of Reproduction and Fertility 55 1-7

Bearer EL and Friend OS (1982) Modifications of anionic-lipid domains preceding membrane fusion in guinea-pig spermatozoa Journal of Cell Biology 92 $604-615$

Brillard JP and McDaniel GR (1985) The reliability and efficiency of various methods for estimating sperm concentration Poultry Science 64 155-158

Cecil HC and Bakst MR (1993) In vitro lipid peroxidation of turkey spermatozoa Poultry Science 72 1370-1378

Chaudhuri D and Wishart GJ (1988) Predicting the fertilising ability of avian semen: the development of an objective colourimetric method for assessing the metabolic activity of fowl spermatozoa British Poultry Science 29 837-845

Christie WW (1982) Isolation of lipids from tissues. In Lipid Analysis: Isolation, Separation, Identification and Structural Analysis of Lipids (2nd Edn) pp 17-25. Pergamon Press, Oxford

Christie WW (1988) Separation of molecular species of triacylglycerols by high-performance liquid chromatography with a silver-ion column Journal of Chromatography 454 273-284.

Christie WW, Noble RC and Moore JH (1970) Determination of lipid classes by gas chromatographic procedure Analyst 95 940-944

Darin-Bennett A, Poulos A and White IG (1974) The phospholipids and phospholipid-bound fatty acids and aldehydes of dog and fowl spermatozoa Journal of Reproduction and Fertility 41 471-474

Elias PM, Friend OS and Georbe J (1979) Membrane sterol heterogeneity: freeze fracture detection with saponin and filipin Journal of Histochemistry and Cytochernistry 27 1247-1260

El Jack MH and Lake PE (1966) The effect of resting roosters from ejaculation on the quality of spermatozoa in semen Journal of Reproduction and Fertility 11 489-491

Evans RW and Setchell BP (1978) Association of exogenous phospholipid with spermatozoa Journal of Reproduction and Fertility $\mathbf{5 3} 357-362$

Froman DP and Thurston RJ (1981) Chicken and turkey spermatozoa superoxide dismutase: a comparative study Biology of Reproduction 24 193-200

Hammerstedt RH (1993) Maintenance of bioenergetic balance in sperm and prevention of lipid peroxidation: a review of the effect on design of storage preservation systems Reproduction. Fertility and Development 5 675-690

Howarth B (1980) The role of lipids in avian sperm survival Proceedings of the 9th International Congress on Animal Reproduction and AI Madrid 2 505-509

Howarth B, Torregrossa D and Britton WM (1977) The phospholipid content of ejaculated fowl and turkey spermatozoa Poultry Science 56 1265-1268

Jain YC and Anand SR (1976) Fatty acids and fatty aldehydes of buffalo seminal plasma and sperm lipid Journal of Reproduction and Fertility 47 261-267

Jones R, Mann T and Sherins R (1979) Peroxidative breakdown of phospholipids in human spermatozoa, spermicidal properties of fatty acid peroxides and protective action of seminal plasma Fertility and Sterility 31 531-537

Lake PE and Stewart JM (1978) AI in poultry Bulletin 213, Ministry of Agriculture, Fisheries and Food, London 
Lin DS, Connor WE, Wolf DP, Neuringer M and Hachey DL (1993) Unique lipids of primate spermatozoa desmosterol and DHA Journal of Lipid Research 34 491-499

Neill AR and Masters CJ (1972) Metabolism of fatty acids by bovine spermatozoa Biochemistry Joumal 127 375-385

Neuringer M, Anderson GJ and Connor WE (1988) The essentiality of n-3 fatty acids for the development and function of the retina and brain Annual Review of Nutrition 8 517-541

Nissen HP and Kreysel HW (1983) Polyunsaturated fatty acids in relation to sperm motility Andrologia 15 264-269

Nissen HP, Kreysel HW and Schirren C (1981) The significance of fatty acids in human spermatozoa and impaired fertility Andrologia 13 444-45

Oliw EH and Sprecher H (1989) Metabolism of polyunsaturated fatty acids by an (n-6) lipoxygenase associated with human ejaculates Biochimica et Biophysica Acta 1002 283-291

Olsen RE and Henderson RJ (1989) The rapid analysis of neutral and polar marine lipids using double-developed HPTLC and scanning densitometry Journal of Experimental Marine Biology and Ecology 129 189-197

Poulos A and White IG (1973) The phospholipid composition of human spermatozoa and seminal plasma Journal of Reproduction and Fertility 35 $265-272$
Poulos A, Darin-Bennett A and White IG (1973) The phospholipid-bound fatty acids and aldehydes of mammalian spermatozoa Comparative Biochemistry and Physiology B 46 541-549

Ravie O and Lake PE (1985) The phospholipid-bound fatty acids of fowl and turkey spermatozoa Animal Reproduction Science 9 189-192

Roldan ERS and Harrison RAP (1993) Diacylglycerol in the exocytosis of the mammalian sperm acrosome Biochemical Society Transactions 21 284-289

Scott TW (1973) Lipid metabolism of spermatozoa Journal of Reproduction and Fertility 18 65-76

Sebastian SM, Selvaraj S, Aruldhas MM and Govindarajulu P (1987) Pattern of neutral and phospholipids in the semen of normospermic, oligospermic and azoospermic men Journal of Reproduction and Fertility 79 373-378

Selley ML, Lacey MJ, Bartlett MR, Copeland CM and Ardlie NG (1991) Content of significant amounts of cytotoxic end-product of lipid peroxidation in human semen Journal of Reproduction and Fertility 92 291-298

Wishart GJ (1984) Effects of lipid peroxide formation in fowl semen on sperm motility, ATP content and fertilizing ability Journal of Reproduction and Fertility 71 113-118

Wishart GJ (1989) Physiological changes in fowl and turkey spermatozoa during in vitro storage British Poultry Science 30 443-454 